\title{
Alterations in Social Brain Network Topology at Rest in Children With Autism Spectrum Disorder
}

\section{Narae Yoon}

Seoul National University Hospital https://orcid.org/0000-0002-5415-4617

\section{Youngmin Huh}

Seoul National University College of Medicine

\section{Hyekyoung Lee}

Seoul National University College of Medicine

Johanna Inhyang Kim

Hanyang University Medical Center

Jung Lee

Seoul National University College of Medicine

\section{Chan-Mo Yang}

Seoul National University College of Medicine

\section{Soomin Jang}

Seoul National University College of Medicine

\section{Yebin D. Ahn}

Seoul National University College of Medicine

\section{Dong Soo Lee}

Seoul National University College of Medicine

\section{Hyejin Kang}

Seoul National University College of Medicine

\section{Bung-Nyun Kim ( $\sim$ kbn1@snu.ac.kr)}

Seoul National University College of Medicine

\section{Research}

Keywords: ASD, functional connectivity, resting state fMRI, graph filtration, graph theory

Posted Date: January 7th, 2022

DOI: https://doi.org/10.21203/rs.3.rs-1206436/v1

License: (c) (i) This work is licensed under a Creative Commons Attribution 4.0 International License. 



\section{Alterations in social brain network topology at rest in children with autism spectrum}

disorder

Narae Yoon ${ }^{1 \dagger}$, Youngmin Huh $^{4 \dagger}$, Hyekyoung Lee ${ }^{2,3}$, Johanna Inhyang Kim ${ }^{6}$, Jung Lee ${ }^{1,7}$, Chan-

Mo Yang ${ }^{1}$, Soomin Jang ${ }^{1}$, Yebin D. Ahn ${ }^{1}$, Dong Soo Lee ${ }^{3,5}$, Hyejin Kang ${ }^{2,3 *}$, Bung-Nyun $\operatorname{Kim}^{1 *}$

${ }^{1}$ Division of Children and Adolescent Psychiatry, Department of Psychiatry, Seoul National University College of Medicine, Seoul, Korea

${ }^{2}$ Biomedical Research Institute, Seoul National University Hospital, Seoul, Korea

${ }^{3}$ Department of Nuclear Medicine, Seoul National University College of Medicine, Seoul, Korea

${ }^{4}$ Medical Research Center, Seoul National University College of Medicine, Seoul, Korea

${ }^{5}$ Department of Molecular Medicine and Biopharmaceutical Science, Seoul National University, Seoul, Korea

${ }^{6}$ Department of Psychiatry, Hanyang University Medical Center, Seoul, Korea

${ }^{7}$ Integrative Care Hub, Seoul National University Children's Hospital, Seoul, Korea 


\section{Corresponding Authors}

*Bung-Nyun Kim

Department of Psychiatry, Seoul National University College of Medicine, 101 Daehakno, Jongno-gu, Seoul 03080, South Korea

Tel: +82-2-2072-3647; Fax: +82-2-747-5774; E-mail: kbn1@snu.ac.kr

*Hyejin Kang

Biomedical Research Institute, Seoul National University Hospital, Seoul, Korea, 101

Daehakno, Jongno-gu, Seoul 03080, South Korea

Tel: +82-2-740-8542; Fax: +82-2-745-7693; E-mail: hkang211@snu.ac.kr 


\begin{abstract}
Background: Underconnectivity in the resting brain is not consistent in autism spectrum disorder (ASD). However, it is known that the default mode network is mainly decreased in childhood ASD. This study investigated the brain network topology as the changes in the connection strength and network efficiency in childhood ASD, including the early developmental stages.
\end{abstract}

Methods: In this study, 31 ASD children aged 2-11 years were compared with 31 age and sex-matched children showing typical development. We explored the functional connectivity based on graph filtration by assessing the single linkage distance and global and nodal efficiencies using resting-state functional magnetic resonance imaging. The relationship between functional connectivity and clinical scores was also analyzed.

Results: Underconnectivities within the posterior default mode network subregions and between the inferior parietal lobule and inferior frontal/superior temporal regions were observed in the ASD group. These areas significantly correlated with the clinical phenotypes. The global, local, and nodal network efficiencies were lower in children with ASD than in those with typical development. In the preschool-age children (2-6 years) with ASD, the anterior-posterior connectivity of the default mode network and cerebellar connectivity were reduced.

Conclusions: The observed topological reorganization, underconnectivity, and disrupted efficiency in the default mode network subregions and social function-related regions could be significant biomarkers of childhood ASD.

Keywords: ASD, functional connectivity, resting state fMRI, graph filtration, graph theory 


\section{Introduction}

Despite the almost unanimous view that biological causes play the most important role in the development of autism spectrum disorders (ASD), the phenomenological diagnostic approach based on core symptoms such as impaired social interaction and restricted and repetitive behaviors is still used. Due to the diagnostic limitations of ASD, the average age of diagnosis is delayed, which not only makes it difficult to intervene early, but also increases the socioeconomic burden. To increase the diagnostic stability and predictability, research is being conducted actively to identify the major neurobiological pathogenesis of ASD.

Although no clear mechanism related to the abnormal brain development in ASD has been identified, the understanding of the neurobiological causes of ASD is gradually increasing, and research on neuroimaging plays an important role. Studies are being actively conducted to extract ASD-specific brain circuits, and functional brain networks using resting-state functional magnetic resonance imaging (rs-fMRI) have been widely used to explore the relationships between brain function and phenotypic features in ASD.

Numerous studies on brain functional connectivity in ASD suggest that it is related to alterations in the connectivity between distinct brain areas, rather than deficits in the function of specific brain regions (1). Altered functional connectivity in ASD has been widely reported through rs-fMRI studies (2), but the findings regarding under- and overconnectivity in the resting brain are inconsistent (3). Several studies have reported underconnectivity in ASD patients, while others have reported overconnectivity (3-5). 
Evidence regarding abnormalities in the functional connectivity in ASD has gradually accumulated, and it has been suggested that changes in the major brain networks such as the default mode network (DMN) are important in the pathogenesis of ASD. Previous studies have reported that the DMN is one of the most informative brain networks for distinguishing between ASD patients and healthy controls $(6,7)$. The DMN is a well-known brain network overlapping with the social brain network, which is activated during social cognition (8). However, the exact mechanisms of the brain networks associated with ASD have not been identified, and majority of the previous studies on the functional connectivity in ASD have targeted patients aged $\geq 7$ years. Since ASD is an early onset disease and brain development is fundamental for disease onset, it is important to explore the brain network connectivity at a younger age. Nevertheless, there are insufficient studies on functional connectivity in young children with ASD.

The results of studies on region of interest (ROI)-based functional connectivity in ASD are inconsistent in terms of individual edges based on the comparison of the correlation matrices. The abnormal functional connectivity in children with ASD through the direct comparison of correlation matrices with children showing typical development (TD) was not robust across different studies. There are several reasons for this inconsistency, such as sample heterogeneity, different network definitions such as nodes and edges, and different network thresholds. We investigated the topological features of a brain network measuring connected components, which correspond to the 0 -th Betti number in persistent homology. We measured the single linkage distance (SLD) to quantify the changes in the connected components by performing graph filtration (9). Graph filtration can bypass the arbitrariness of thresholding for network construction. The SLD for identifying the topological features of 
the brain network was investigated in ASD, attention deficit hyperactivity disorder $(9,10)$, and Parkinson disease (11).

This study aimed to identify and compare the abnormal topological features of functional connectivity using rs-fMRI between children with ASD and those with TD. We used the graph filtration approach to investigate the topological features of the brain network and a graph-theoretical approach to explore the local and nodal efficiencies of the whole brain network. We hypothesized that the following: 1) children with ASD show atypical functional connectivity across the whole brain network and within the DMN regions as compared to the age and sex-matched TD children and 2) atypical functional connectivity and clinical features are significantly correlated. 


\section{Materials and methods}

\section{Participants}

This study included 31 children with ASD and 31 age- and sex-matched children with TD. All the ASD patients were initially screened by board-certified psychiatrists for children and adolescents based on the diagnostic criteria of the Diagnostic and Statistical Manual, 5th edition. They were diagnosed according to the Autism Diagnostic Observation Schedule (ADOS) (12) and Korean Childhood Autism Rating Scale, second edition (KCARS-2) (13) at the Child and Adolescent Psychiatry Clinic in Seoul National University Hospital. The exclusion criteria for the ASD group were hereditary genetic disorder, current or past history of brain trauma, organic brain disorder, seizure, or any neurological disorder, schizophrenia or any other childhood-onset psychotic disorder, major depressive disorder or bipolar disorder, Tourette's syndrome or a chronic motor/vocal tic disorder, and obsessivecompulsive disorder. The TD group included typically developing children without any psychiatric diagnoses. The exclusion criteria of the ASD group were also applied to the TD group with the additional exclusion criterion of ASD diagnosis.

\section{Clinical measures}

To examine intelligence, we used the Korean Educational Development InstituteWechsler Intelligence Scale for Children-Revised (14) for school-age children with language comprehension and the Korean-Leiter International Performance Scale-Revised (15) for preschool- and school-age children without language comprehension. Social ability and social competence were assessed using the Korean translated version of the Social 
Responsiveness Scale (SRS) (16) and the Social Maturity Scale (17). To identify autistic properties, the K-CARS-2 was used for both the ASD and TD groups.

\section{Image acquisition and processing}

\section{Image acquisition}

All participants underwent high-resolution T1-weighted structural imaging, diffusion tensor imaging (DTI), and rs-fMRI. Scanning was performed using Siemens Trio 3 T MRI scanner (Siemens Medical Systems, Erlangen, Germany) at the Seoul National University Hospital. The images were acquired with the following parameters: repetition time, $3000 \mathrm{~ms}$; echo time, $40 \mathrm{~ms}$; flip angle, $90^{\circ}$; voxel size, $1.9 \times 1.9 \times 4.0 \mathrm{~mm}^{3}$; field of view, $240 \mathrm{~mm}$; slice number, 35 (interleaved); matrix, $128 \times 128$ (or $64 \times 64$ ); and slice thickness, $4.0 \mathrm{~mm}$. The entire MRI protocol lasted $35 \mathrm{~min}$ and consisted of structural MRI, DTI, and rs-fMRI. We only analyzed the rs-fMRI data, as the purpose of this study was to explore the differences in the brain functional connectivity between the ASD patients and TD children.

The safety of MRI was confirmed prior to the scan for each participant and their parents using checklists. During the scan, the head of each participant was fixed using cushions without causing them any discomfort, and the entire scanning process was monitored by a certified psychiatrist and the parent.

Some of the participants needed to be sedated with oral chloral hydrate to ensure immobility during MRI. The prescription and administration of the drug were performed carefully by certified psychiatrists, and doses considered safe for children (25-50 mg/kg; maximum dose, $1 \mathrm{~g}$ ) were administered after the consent of the parent/guardian and patient. 
Additionally, the medical staff monitored the patient's condition until they recovered fully. Oral chloral hydrate was administered for sedation in 29 ASD children and 15 TD children in this study, and majority of the children with TD who underwent sedation were 2-6 years old. Therefore, we additionally analyzed the functional connectivity under sedation between 15 ASD and 15 TD children of the preschool age group.

\section{Image preprocessing}

Echo planar images were preprocessed using Statistical Parametric Mapping (www.fil.ion.ucl.ac.uk/spm/). The first four images were discarded due to magnetic stabilization. The motion parameters were estimated with respect to the first image. Despiking was performed using BrainWavelet Toolbox (https://www.brainwavelet.org/) (18). The slice time was adjusted to the middle of the repetition time. The data were realigned by rigid body transformation. For the registration to the standard space, the anatomical image was normalized to the National Institute of Health's Pediatric Database Objective 1 atlas (4.5-18.5 years age range, asymmetric template) after co-registration of anatomical and mean functional images $(19,20)$. After smoothing using Gaussian kernel of 6-mm full width at half maximum and intensity normalization to the whole brain median of 1000 (18), six motion parameters and white matter/cerebrospinal fluid signal were regressed out. Finally, bandpass filtering $(0.01-0.1 \mathrm{~Hz})$ was performed. We generated a functional brain network consisting of 246 cortical ROIs using Brainnetome atlas (21) and 28 cerebellar regions of SUIT (http://www.diedrichsenlab.org/imaging/suit.htm) (22). One ROI, vermis_crus-I, was excluded because of its small size. A total of 273 ROIs were considered as the network nodes. The connectivity matrices for each participant were calculated using Pearson's correlation 
coefficient.

\section{Graph filtration}

To investigate the topological features of the brain network with varying thresholds, we applied graph filtration to find the connected components of the brain graph with filtration. The shape of the network is represented as a single linkage hierarchical clustering and transformed into an algebraic form, a single linkage matrix (SLM) (9). The SLM was generated by calculating the SLD of $C_{i j}(9) . C_{i j}$ is the correlation coefficient between nodes $i$ and $j$, and only positive correlation was analyzed in this study. The distance between two nodes was calculated to estimate SLD as follows:

Distance $=\sqrt{\left(1-C_{i j}\right)}$

The distance for the threshold gradually increased and was iteratively applied to the distance matrix. The SLM enables the comparison of the patterns of the connecting components between the two groups. Nodes with a shorter distance than the threshold were identified as connected components, and shorter distance between nodes reflects closer coupling between nodes.

\section{Network efficiencies}

To investigate the efficiency of the brain network, we calculated global (Eglobal), local (Elocal) and nodal efficiencies (Enodal) using Brain Connectivity Toolbox (https://sites.google.com/site/bctnet/) (23). A graph $G(N, K)$ comprising $N$ nodes and $K$ edges 
and a binary matrix, $A_{i j}$, can be represented by a $273 \times 273$ matrix, where $A_{i j}=\left\{\begin{array}{l}1, \text { if there is an edge with positive value between node } i \text { and } j \\ 0, \text { otherwise }\end{array}\right.$

Degree of $i, \mathrm{~K}_{i}$, is the number of connections linking the node $i$ :

$$
k_{i}=\sum_{j \in G} A_{i j}
$$

Global efficiency (Eglobal) of graph $G$ is defined as:

$$
E_{\text {global }}(G)=\frac{1}{N(N 1)} \sum_{i \neq j \in G} \frac{1}{L_{i j}}
$$

where, $L_{i j}$ is the shortest path length between nodes $i$ and $j$ in the weighted network $W$, and it is defined as the smallest sum of the edge distance equivalent to weight inversion throughout all possible paths between nodes $i$ and $j$. Global efficiency measures the efficiency of parallel information propagation over the network. The local efficiency (Elocal) of a node is calculated as:

$$
E_{\text {local }}(\mathrm{i})=\frac{1}{N} \sum_{i \in G} E_{\text {global }}\left(G_{i}\right)
$$

Where, $G_{i}$ is the subgraph of $G$ and consists of the neighbors of node $i$, and $E_{\text {global }}\left(G_{i}\right)$ denotes the global efficiency of the subgraph $G_{i}$. It measures the fault tolerance of a network, indicating communication capability among the neighbors of node $i$ when it is eliminated. 
Nodal efficiency ( $\left.E_{\text {nodal }}\right)$ measures the efficiency of parallel information transfer across the whole network in a node using the following equation (24):

$$
E_{\text {nodal }}(i)=\frac{1}{N-1} \sum_{i \neq j \in G} \frac{1}{L_{i j}}
$$

\section{Statistical analysis}

In this study, the following four measures were statistically tested: SLD $\mathrm{ij}_{\mathrm{j}}, \mathrm{E}_{\mathrm{global}}$, $E_{\text {local }}(i)$, and $E_{\text {nodal }}(i)$. The linear regression model evaluated differences in SLD and network measures between the two groups. We included age and sex in the model to regress out the effects as follows:

$$
y=\beta_{0}+\beta_{1} \times \text { Age }+\beta_{2} \times \operatorname{Sex}+\beta_{3} \times \text { Group }+e
$$

where, $y$ represents the four measures, $S L D_{i j}, E_{\text {global }}, E_{\text {local }}(i)$, and $E_{\text {nodal }}(i)$, and the fitting was implemented using the least-squares method. $\beta$ denotes estimated coefficients of the variables (age, sex, and group), and $e$ refers to an error term. We investigated significant group effects (SLD: $\mathrm{p}<0.00005$, uncorrected; network measure: $\mathrm{p}<$ 0.05 , false discovery rate [FDR] corrected) to find differences between the groups.

To evaluate correlations between measures and age within a group, we performed Pearson's correlation after partialling out of the sex effect using linear regression.

Next, we investigated the association between measures and clinical scores in each of the two groups by Spearman's correlation after regressing out age and sex effects using a linear regression model. We tested the correlation between edges that showed significant group 
differences and 11 clinical scores: CARS, ADOS communications, ADOS social interaction, ADOS total, SRS social awareness, SRS social cognition, SRS communication, SRS social motivation, SRS restricted interests and repetitive behavior, SRS social communications index, and SRS total. We analyzed edges/nodes that showed a significant correlation with clinical scores $(p<0.05)$. We analyzed the group differences in the SLD in preschool-age children (age, 2-6 years; ASD group, $n=15$ and TD group, $n=15$ ) to determine whether the underconnectivity was due to the sedation effect. All the preschool-age children in both the groups were sedated. 
Table 1. Demographic and clinical characteristics

\begin{tabular}{|c|c|c|c|c|c|c|c|c|}
\hline & \multirow{3}{*}{ Variables } & \multicolumn{6}{|c|}{ Groups } & \multirow{3}{*}{$\begin{array}{c}p- \\
\text { value }\end{array}$} \\
\hline & & \multicolumn{3}{|c|}{$\operatorname{ASD}(n=31)$} & \multicolumn{3}{|c|}{ TD $(n=31)$} & \\
\hline & & Mean & SD & $\mathbf{n}$ & Mean & SD & $\mathbf{n}$ & \\
\hline $\begin{array}{l}\text { Age } \\
\text { (years) }\end{array}$ & & 5.61 & 2.53 & 31 & 5.65 & 2.48 & 31 & \\
\hline \multirow[t]{2}{*}{ Sex } & Boy & & & 19 & & & 19 & \\
\hline & Girl & & & 12 & & & 12 & \\
\hline Sedation & & & & 30 & & & 15 & \\
\hline \multicolumn{9}{|l|}{ ADOS } \\
\hline & Communication & 5.56 & 1.45 & 27 & - & - & - & \\
\hline & Social interaction & 8.93 & 2.51 & 27 & - & - & - & \\
\hline & Total & 14.56 & 3.62 & 27 & - & - & - & \\
\hline \multicolumn{9}{|l|}{ CARS } \\
\hline & & 29.86 & 6.29 & 29 & 15.52 & 0.73 & 28 & $<000$ \\
\hline \multicolumn{9}{|l|}{ SRS } \\
\hline & Awareness & 11.94 & 3.58 & 17 & 5.95 & 3.02 & 21 & $<000$ \\
\hline & Cognition & 20.00 & 5.10 & 17 & 7.05 & 3.25 & 21 & $<000$ \\
\hline & Communication & 33.18 & 9.37 & 17 & 7.62 & 4.54 & 21 & $<000$ \\
\hline & Motivation & 12.88 & 5.94 & 17 & 5.43 & 3.60 & 21 & $<000$ \\
\hline & Responsiveness & 13.59 & 5.73 & 17 & 3.81 & 3.46 & 21 & $<000$ \\
\hline & $\begin{array}{l}\text { Social communication } \\
\text { index }\end{array}$ & 78.00 & 21.44 & 17 & 26.05 & 11.75 & 21 & $<000$ \\
\hline & Total & 91.59 & 26.54 & 17 & 29.86 & 13.57 & 21 & $<000$ \\
\hline
\end{tabular}

SD, standard deviation; ADOS, Autism Diagnostic Observation Schedule; CARS, Childhood Autism Rating Scale; SRS,

Social Responsiveness Scale; ASD group, children with autism spectrum disorder; TD group, children with typical

development 


\section{Results}

\section{$4 \quad$ Group differences in network topology based on graph filtration}

5 To find significant group differences in network topology based on graph filtration, we

6 compared the SLDs between the two groups. Increased SLDs were found in the ASD group

$7 \quad(p<0.01$, FDR corrected). A longer SLD denotes weaker functional connectivity. In ASD, the

8 bilateral connectivities of the dorsal part of the cingulate gyri (area 23) and precuneus (area

9 31) were significantly decreased, which are sub-regions of the DMN. The bilateral

10 connectivity between the dorsal part of the cingulate gyri significantly correlated with the behavioral score, i.e., the CARS ( $r h o=0.5, p<0.05 ; \mathrm{n}=29)$ and SRS communication $(r h o=$ $0.68, p<0.005 ; \mathrm{n}=17$ ) scores, in the ASD group (Figure 1 and Table 2). As compared to the TD group, the ASD group showed decreased functional connectivity of the right inferior parietal lobule (IPL) (rostroventral area 40) with the left inferior frontal gyrus (IFG) (caudal area 45). Moreover, the functional connectivity in the ASD group significantly correlated with the sub-scores of the SRS for communication ( $r h o=0.68, p<0.005 ; \mathrm{n}=17$ ), awareness $(r h o=0.52, p<0.03 ; \mathrm{n}=17)$, and social communication index $(r h o=0.52, p<0.03 ; \mathrm{n}=17)$. The left IPL was significantly connected with the right superior frontal gyrus (SFG) (medial area 9), left IFG (caudal area 45), right superior temporal gyrus (STG) (rostral area 22), and left inferior temporal gyrus (ITG) (ventrolateral area 37) (Figure 1 and Table 2). The functional distance between the left IPL (rostroventral area 40) and left IFG positively correlated with the SRS communication score $(r h o=0.54, p<0.02 ; \mathrm{n}=17)$ in the ASD group. 


\begin{tabular}{|c|c|c|c|c|c|c|c|c|c|}
\hline \multicolumn{3}{|c|}{ Connectivity } & \multicolumn{7}{|c|}{ Clinical correlation } \\
\hline \multirow[b]{2}{*}{ ROI 1} & \multirow[b]{2}{*}{ ROI 2} & \multirow{2}{*}{$\begin{array}{c}p \text { - } \\
\text { value } \\
\text { (FDR) }\end{array}$} & & \multicolumn{3}{|c|}{ ASD group } & \multicolumn{3}{|c|}{ TD group } \\
\hline & & & & rho & $p$ & $\%$ sub & rho & $p$ & $\%$ sub \\
\hline \multirow[t]{2}{*}{ Right, Cingulate, dorsal area 23} & \multirow[t]{2}{*}{ Left, Cingulate, dorsal area 23} & \multirow[t]{2}{*}{$<0.01$} & CARS & 0.45 & 0.02 & 93.55 & 0.40 & 0.03 & 93.55 \\
\hline & & & SRS Com & 0.68 & 0.00 & 54.84 & 0.00 & 1.00 & 54.84 \\
\hline Right, Precuneus, area 31 & Left, Precuneus, are 31 & $<0.01$ & & & & & & & \\
\hline \multirow[t]{3}{*}{$\begin{array}{l}\text { Right, Inferior parietal lobule, } \\
\text { rostroventral area } 40\end{array}$} & \multirow[t]{3}{*}{$\begin{array}{l}\text { Left, Inferior frontal gyrus, caudal } \\
\text { area } 45\end{array}$} & $<0.01$ & SRS Com & 0.68 & 0.00 & 54.84 & 0.14 & 0.59 & 54.84 \\
\hline & & & SRS Awa & 0.52 & 0.03 & 54.84 & 0.27 & 0.29 & 54.84 \\
\hline & & & SCI & 0.52 & 0.03 & 54.84 & 0.19 & 0.47 & 54.84 \\
\hline \multirow[t]{4}{*}{$\begin{array}{l}\text { Left, Inferior parietal lobule, } \\
\text { rostroventral area } 40\end{array}$} & $\begin{array}{l}\text { Right, Superior frontal gyrus, medial } \\
\text { area } 9\end{array}$ & $<0.01$ & & \multirow{4}{*}{0.54} & \multirow{4}{*}{0.02} & \multirow{4}{*}{54.84} & \multirow{4}{*}{0.00} & \multirow{4}{*}{0.99} & \multirow{4}{*}{54.84} \\
\hline & $\begin{array}{l}\text { Left, Inferior frontal gyrus, caudal } \\
\text { area } 45\end{array}$ & $<0.01$ & \multirow[t]{3}{*}{ SRS Com } & & & & & & \\
\hline & $\begin{array}{l}\text { Right, Superior temporal gyrus, } \\
\text { rostral area } 22\end{array}$ & $<0.01$ & & & & & & & \\
\hline & $\begin{array}{l}\text { Left, Inferior temporal gyrus, } \\
\text { ventrolateral area } 37\end{array}$ & $<0.01$ & & & & & & & \\
\hline
\end{tabular}

25 ROI, region of interest; FDR, false discovery rate; CARS, Childhood Autism Rating Scale; SRS, Social Responsiveness Scale; com; communication; Awa; social

26 awareness; SCI, Social Communications Index; ASD group, children with autism spectrum disorder; TD group, children with typical development; \% sub, the percentage

27 of subjects who have clinical score 

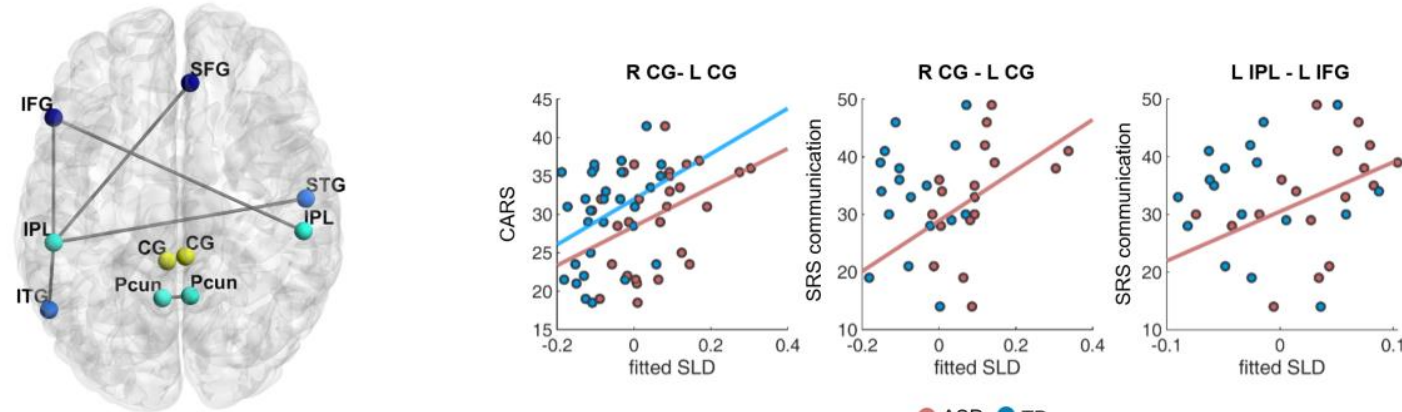

ASD $O$ TD
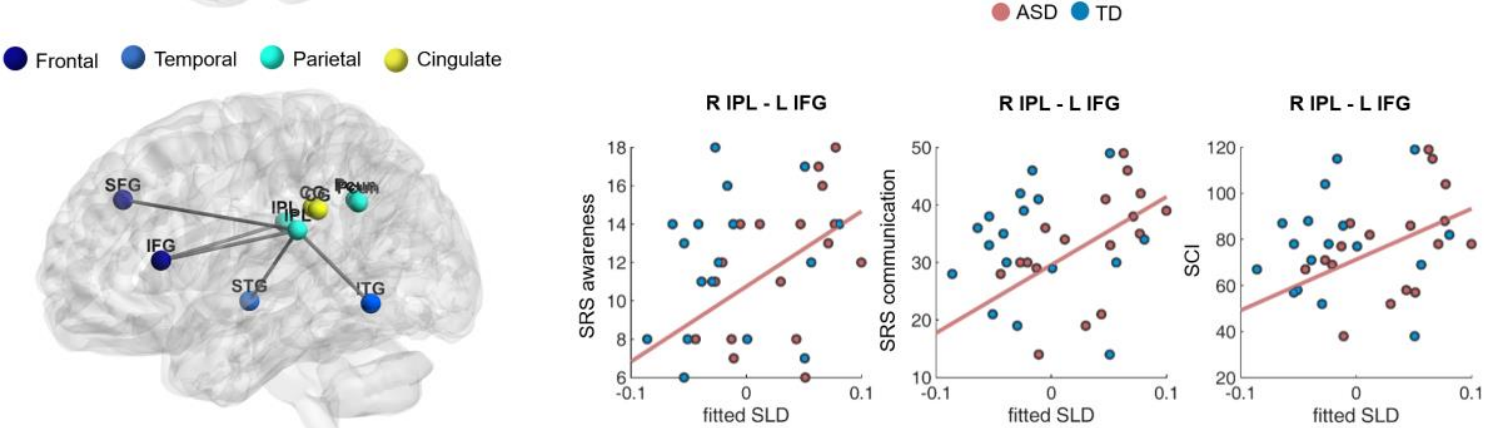

Fig. 1 Edges with significantly decreased functional connectivity and their correlations with clinical scores.

Edges with significantly increased SLD are shown ( $p<0.01$, FDR corrected) (top). The children with ASD showed increased SLD between the bilateral dorsal parts of the cingulate gyri (area 23) and between bilateral precuneus (area 31). Edges with increased SLD were also observed between the IPL and the frontal/temporal regions. Significant correlations were found between edges showing increased SLD and the clinical scores $(p<0.05)$. The SLD between the right IPL and left IFG positively correlated with the SRS communication, SRS social awareness, and SCI scores. The SLD between the left IPL and the left IFG positively correlated with the SRS communication score. Additionally, there was a positive correlation between the SLD of an edge connecting the bilateral cingulate gyrus and the clinical scores of CARS and SRS communication (bottom, $p<0.05$ ). 
42 (SLD, single linkage distance; FDR, false discovery rate; SRS, Social Responsiveness Scale;

43 SCI, Social Communication Index; SRS communication, Social Responsiveness Scale

44 communication; SRS awareness, Social Responsiveness Scale social awareness; CARS,

45 Childhood Autism Rating Scale; R, right; L, left; CG, cingulate gyrus; IPL, inferior parietal

46 lobule; IFG, inferior frontal gyrus; SFG, superior frontal gyrus; Pcun, precuneus; ITG,

47 inferior temporal gyrus; STG, superior temporal gyrus) 

To identify the general network characteristics using graph theoretical measures, we examined the network efficiencies, comprising global efficiency, local efficiency, and nodal 51 efficiency. Global efficiency significantly decreased $(p<0.005)$ in the ASD group (Figure 2).

52 The local efficiency of the left IFG and subgenual area 32 of the cingulate gyrus was lower in the ASD group than in the TD group ( $p<0.05$, FDR corrected) (Table 3 and Figure 3 ). The local efficiency of the cingulate gyrus negatively correlated with the ADOS total $(r h o=$ $-0.45, p<0.02$ ) and ADOS social interaction (rho $=-0.53, p<0.005)$ scores (Table 4). The ASD group showed decreased nodal efficiency in the frontal, parietal, insula, and occipital areas as compared to the TD group ( $p<0.05$, FDR corrected) (Table 3 and Figure 3$)$. The nodal efficiencies of the parietal and occipital areas negatively correlated with the CARS scores in the ASD group. The nodal efficiencies of the frontal and parietal areas negatively correlated and the cerebellar crus positively correlated with the ADOS sub-scores. The nodal efficiencies of the occipital and temporal areas negatively correlated with the SRS sub-scores in the ASD group (Table 4). 


\begin{tabular}{|c|c|c|}
\hline $\begin{array}{l}\text { Network } \\
\text { measure }\end{array}$ & ROI & $\begin{array}{l}p \text {-value } \\
\text { (FDR } \\
\text { corrected) }\end{array}$ \\
\hline \multicolumn{3}{|l|}{ Local efficiency } \\
\hline & Left, Cingulate gyrus, subgenual area 32 & $\begin{array}{l}<0.05 \\
\text { FWER }\end{array}$ \\
\hline & Left, Inferior frontal gyrus, caudal area 45 & $\begin{array}{l}<0.05, \\
\text { FWER }\end{array}$ \\
\hline \multicolumn{3}{|l|}{ Nodal efficiency } \\
\hline \multirow[t]{20}{*}{ Frontal lobe } & Right, Superior frontal gyrus, dorsolateral area 8 & $<0.01$ \\
\hline & Right, Superior frontal gyrus, lateral area 9 & $<0.05$ \\
\hline & Right, Superior frontal gyrus, medial area 9 & $<0.05$ \\
\hline & Right, Superior frontal gyrus, medial area 10 & $<0.05$ \\
\hline & Left, Superior frontal gyrus, medial area 10 & $<0.05$ \\
\hline & Left, Superior frontal gyrus, medial area 9 & $<0.05$ \\
\hline & Right, Middle frontal gyrus, inferior frontal junction & $<0.05$ \\
\hline & Right, Middle frontal gyrus ventral area 9/46 & $<0.05$ \\
\hline & Left, Middle frontal gyrus ventral area $9 / 46$ & $<0.05$ \\
\hline & Right, Inferior frontal gyrus, inferior frontal sulcus & $<0.05$ \\
\hline & Right, Inferior frontal gyrus, caudal area 45 & $<0.05$ \\
\hline & Right, Inferior frontal gyrus, ventral area 44 & $<0.01$ \\
\hline & Left, Inferior frontal gyrus, ventral area 44 & $<0.05$ \\
\hline & Left, Inferior frontal gyrus, caudal area 45 & $<0.05$ FWER \\
\hline & Right, Precentral gyrus, area 4 (head and face region) & $<0.01$ \\
\hline & Right, Precentral gyrus, area 4 (upper limb region) & $<0.01$ \\
\hline & Right, Precentral gyrus, area 4 (tongue and larynx region) & $<0.01$ \\
\hline & Right, Precentral gyrus, caudal ventrolateral area 6 & $<0.05$ \\
\hline & Left, Precentral gyrus, caudal ventrolateral area 6 & $<0.05$ \\
\hline & Left, Precentral gyrus, area 4 (tongue and larynx region) & $<0.05$ \\
\hline \multirow{6}{*}{$\begin{array}{c}\text { Temporal } \\
\text { lobe }\end{array}$} & Right, Superior temporal gyrus, area $41 / 42$ & $<0.01$ \\
\hline & Right, Superior temporal gyrus, caudal area 22 & $<0.05$ \\
\hline & Right, Superior temporal gyrus, rostral area 22 & $<0.05$ \\
\hline & Left, Middle temporal gyrus, dorsolateral area 37 & $<0.05$ \\
\hline & Left, Inferior temporal gyrus, caudolateral of area 20 & $<0.05$ \\
\hline & Right, Superior temporal sulcus, rostroposterior & $<0.05$ \\
\hline \multirow[t]{15}{*}{ Parietal lobe } & Right, Superior parietal lobule, rostral area 7 & $<0.05$ \\
\hline & Right, Superior parietal lobule, caudal area 7 & $<0.01$ \\
\hline & Right, Superior parietal lobule, lateral area 5 & $<0.05$ FWER \\
\hline & Right, Superior parietal lobule, postcentral area 7 & $<0.05$ FWER \\
\hline & Right, Superior parietal lobule, intraparietal area 7 & $<0.01$ \\
\hline & Left, Superior parietal lobule, intraparietal area 7 & $<0.05$ FWER \\
\hline & Left, Superior parietal lobule, postcentral area 7 & $<0.01$ \\
\hline & Left, Superior parietal lobule, lateral area 5 & $<0.05$ FWER \\
\hline & Left, Superior parietal lobule, caudal area 7 & $<0.05$ FWER \\
\hline & Left, Superior parietal lobule, rostral area 7 & $<0.01$ \\
\hline & Right, Inferior parietal lobule, rostrodorsal area 39 & $<0.05$ \\
\hline & Right, Inferior parietal lobule, rostrodorsal area 40 & $<0.01$ \\
\hline & Right, Inferior parietal lobule, rostroventral area 40 & $<0.01$ \\
\hline & Left, Inferior parietal lobule, rostroventral area 40 & $<0.01$ \\
\hline & Left, Inferior parietal lobule, rostroventral area 39 & $<0.01$ \\
\hline
\end{tabular}


Left, Inferior parietal lobule, rostrodorsal area $40 \quad<0.05$

Right, Precuneus, medial area $7 \quad<0.05$

Right, Precuneus, area $31 \quad<0.01$

Left, Precuneus, area $31 \quad<0.05$

Left, Precuneus, medial area $7 \quad<0.05$

Right, Postcentral gyrus, area 1/2/3 (upper limb, head and face $\quad<0.05$

region)

Right, Postcentral gyrus, area 1/2/3 (tongue and larynx region) $\quad<0.05$ FWER

Right, Postcentral gyrus, area $2 \quad<0.05$

Right, Postcentral gyrus, area 1/2/3 (trunk region) $\quad<0.05$

Left, Postcentral gyrus, area $2 \quad<0.01$

Left, Postcentral gyrus, area 1/2/3 (tongue and larynx region) $\quad<0.01$

Left, Postcentral gyrus, area 1/2/3 (upper limb, head and face $\quad<0.05$

Insula $\quad$ Right, Insula, hypergranular insula $<0.05$

Right, Insula, dorsal granular insula $\quad<0.01$

Left, Insula, dorsal granular insula $\quad<0.05$

Left, Hypergranular insula $\quad<0.05$

Cingulate Right, Cingulate gyrus, dorsal area $23<0.05$

Right, Cingulate gyrus, subgenual area $32 \quad<0.05$

Left, Cingulate gyrus, subgenual area $32 \quad<0.05$

Occipital Right, Lateral occipital, medial superior occipital gyrus $\quad<0.05$

Right, Lateral occipital, lateral superior occipital gyrus $\quad<0.05$ FWER

Left, Lateral occipital, lateral superior occipital gyrus $\quad<0.05$ FWER

Left, Lateral occipital, medial superior occipital gyrus $\quad<0.05$

Cerebellum Cerebellar crus II

$<0.05$

65 ROI, region of interest; FDR, false discovery rate; FWER, family-wise error rate; 


\begin{tabular}{|c|c|c|c|c|c|c|c|c|}
\hline \multirow{2}{*}{$\begin{array}{l}\text { Network } \\
\text { measure }\end{array}$} & \multirow[b]{2}{*}{$\begin{array}{l}\text { Clinical } \\
\text { score }\end{array}$} & \multirow{2}{*}{$\begin{array}{c}\text { Significant node } \\
\text { ROI }\end{array}$} & \multicolumn{3}{|c|}{ ASD group } & \multicolumn{3}{|c|}{ TD group } \\
\hline & & & rho & $\begin{array}{c}p- \\
\text { value }\end{array}$ & $\%$ sub & rho & $\begin{array}{c}p- \\
\text { value }\end{array}$ & $\%$ sub \\
\hline \multirow[t]{3}{*}{$\begin{array}{l}\text { Local } \\
\text { efficiency }\end{array}$} & $\begin{array}{l}\text { ADOS } \\
\text { total }\end{array}$ & & & & & & & \\
\hline & ADOS soci & $\begin{array}{l}\text { Left, Cingulate gyrus, subgenual area } 32 \\
\text { ll interaction }\end{array}$ & -0.45 & 0.02 & 87.10 & & & \\
\hline & & Left, Cingulate gyrus, subgenual area 32 & -0.53 & 0.00 & 87.10 & - & - & - \\
\hline \multicolumn{9}{|l|}{$\begin{array}{l}\text { Nodal } \\
\text { efficiency }\end{array}$} \\
\hline & & Left, Superior parietal lobule, lateral area 5 & -0.42 & 0.02 & 93.55 & 0.23 & 0.23 & 90.32 \\
\hline & & Left, Superior parietal lobule, intraparietal area 7 & -0.42 & 0.02 & 93.55 & 0.19 & 0.33 & 90.32 \\
\hline & & Left, Precuneus, medial area 7 & -0.55 & 0.00 & 93.55 & 0.18 & 0.36 & 90.32 \\
\hline & & Left, Lateral superior occipital gyrus & -0.38 & 0.04 & 93.55 & 0.14 & 0.49 & 90.32 \\
\hline & & Right, Inferior parietal lobule, rostrodorsal area 40 & -0.41 & 0.03 & 93.55 & -0.06 & 0.78 & 90.32 \\
\hline & ADOS, con & munications & & & & & & \\
\hline & & Left, Cerebellar crus II & 0.54 & 0.00 & 87.10 & - & - & - \\
\hline & ADOS soci & linteraction & & & & & & \\
\hline & & Left, Cingulate gyrus, subgenual area 32 & -0.57 & 0.00 & 87.10 & - & - & - \\
\hline & & Left, Precuneus, medial area 7 & -0.50 & 0.01 & 87.10 & - & - & - \\
\hline & & Right, Superior frontal gyrus, lateral area 9 & -0.40 & 0.04 & 87.10 & - & - & - \\
\hline & & Right, Superior frontal gyrus, medial area 10 & -0.40 & 0.04 & 87.10 & - & - & - \\
\hline & & Left, Superior frontal gyrus, medial area 9 & -0.40 & 0.04 & 87.10 & - & - & - \\
\hline & $\begin{array}{l}\text { ADOS } \\
\text { total }\end{array}$ & & & & & & & \\
\hline & & Left, Cingulate gyrus, subgenual area 32 & -0.52 & 0.01 & 87.10 & - & - & - \\
\hline & & Left, Precuneus, medial area 7 & -0.47 & 0.01 & 87.10 & - & - & - \\
\hline & & Left, Inferior parietal lobule, rostrodorsal area 40 & -0.41 & 0.03 & 87.10 & - & - & - \\
\hline & & Right, Superior frontal gyrus, medial area 10 & -0.39 & 0.04 & 87.10 & - & - & - \\
\hline & SRS Comn & unication & & & & & & \\
\hline & & Left, Lateral superior occipital gyrus & -0.58 & 0.01 & 54.84 & -0.27 & 0.23 & 67.74 \\
\hline & & $\begin{array}{l}\text { Left, Inferior temporal gyrus, caudolateral of area } \\
20\end{array}$ & -0.57 & 0.02 & 54.84 & 0.10 & 0.68 & 67.74 \\
\hline
\end{tabular}


SRS

Cognition

Right, Lateral superior occipital gyrus

\begin{tabular}{cccccc}
-0.51 & 0.04 & 54.84 & -0.27 & 0.23 & 67.74 \\
& & & & & \\
-0.52 & 0.03 & 54.84 & -0.46 & 0.04 & 67.74 \\
-0.50 & 0.04 & 54.84 & 0.04 & 0.85 & 67.74 \\
-0.49 & 0.04 & 54.84 & -0.30 & 0.19 & 67.74 \\
& & & & \\
-0.48 & 0.05 & 54.84 & -0.37 & 0.10 & 67.74 \\
\hline
\end{tabular}

Right, Precentral gyrus, area 4 (upper limb region)

Right, Lateral superior occipital gyrus

$S C I$

Left, Lateral superior occipital gyrus

D group, children with autism spectrum

68 disorder; TD group, children with typical development; SCI, Social Communications Index 


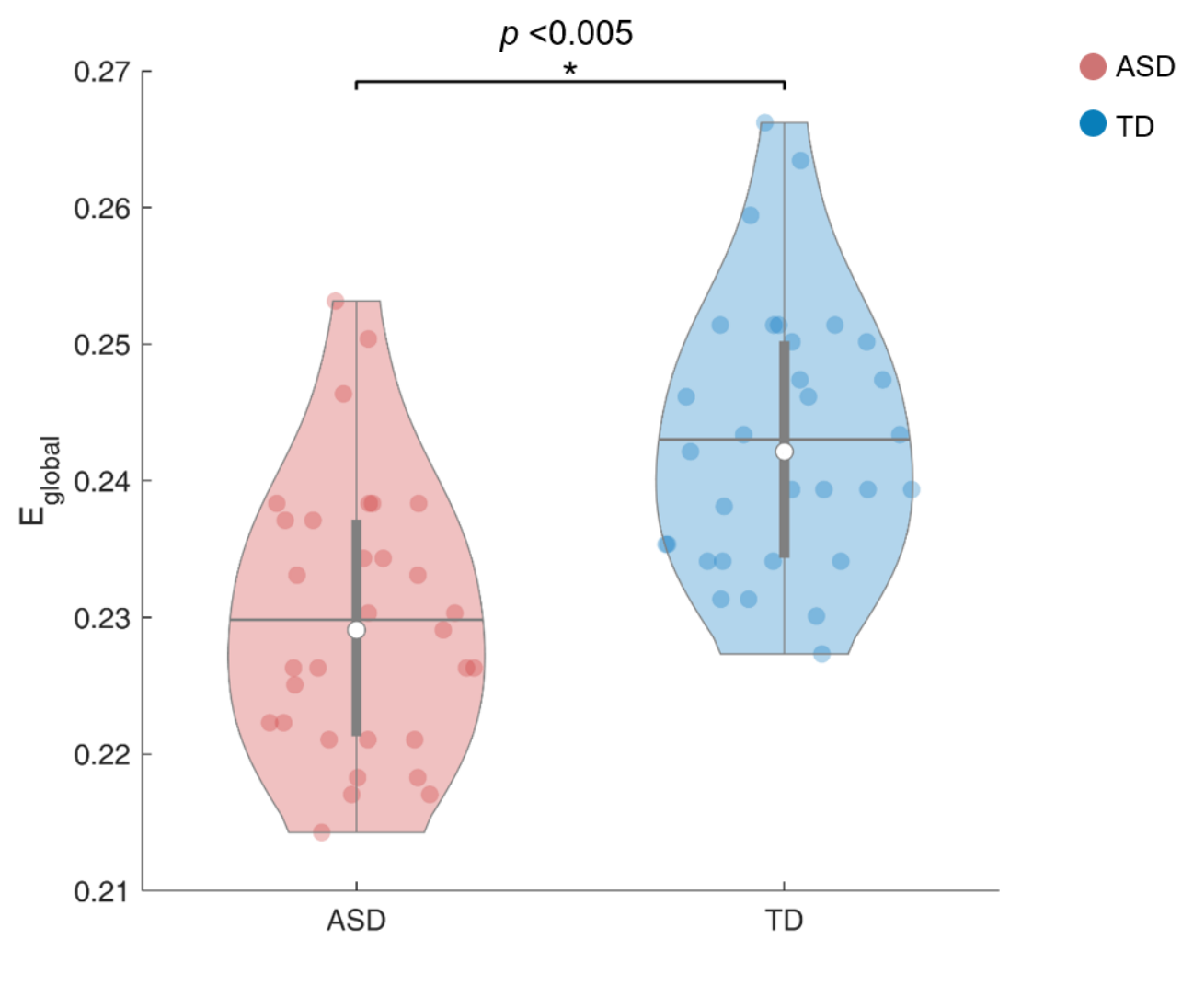

70 Fig. 2 The global efficiency. The ASD group showed significantly decreased global

71 efficiency as compared to the TD group $(p<0.005)$. Violin plots of the ASD and TD groups

72 are shown in red and blue, respectively. The individual circle denotes the global efficiency of

73 each participant (red: ASD; blue: TD). The group average is shown as a gray horizontal line,

74 and white circles represent the median of each group. The filled gray box in the center of

75 each group represents the middle $50 \%$, i.e., the range between the 25 th and 75 th percentiles.

76 (ASD group, children with autism spectrum disorder; TD group, children with typical

77 development; Eglobal, global efficiency)

$78 *$ The significant difference was found at $p<0.005$. 
A)
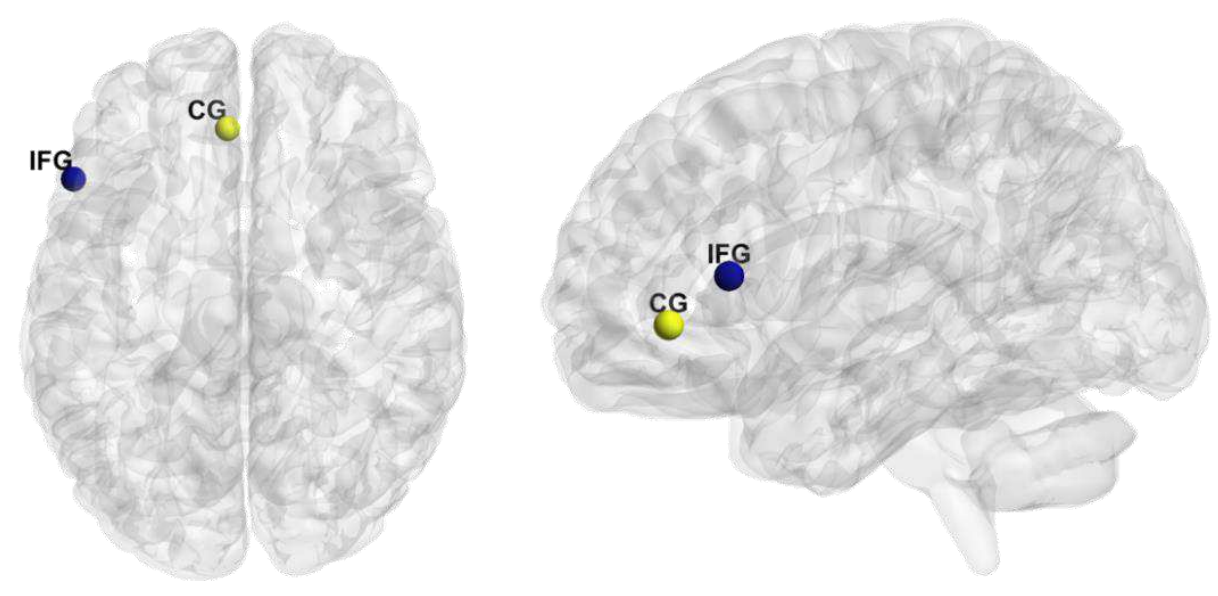

B)
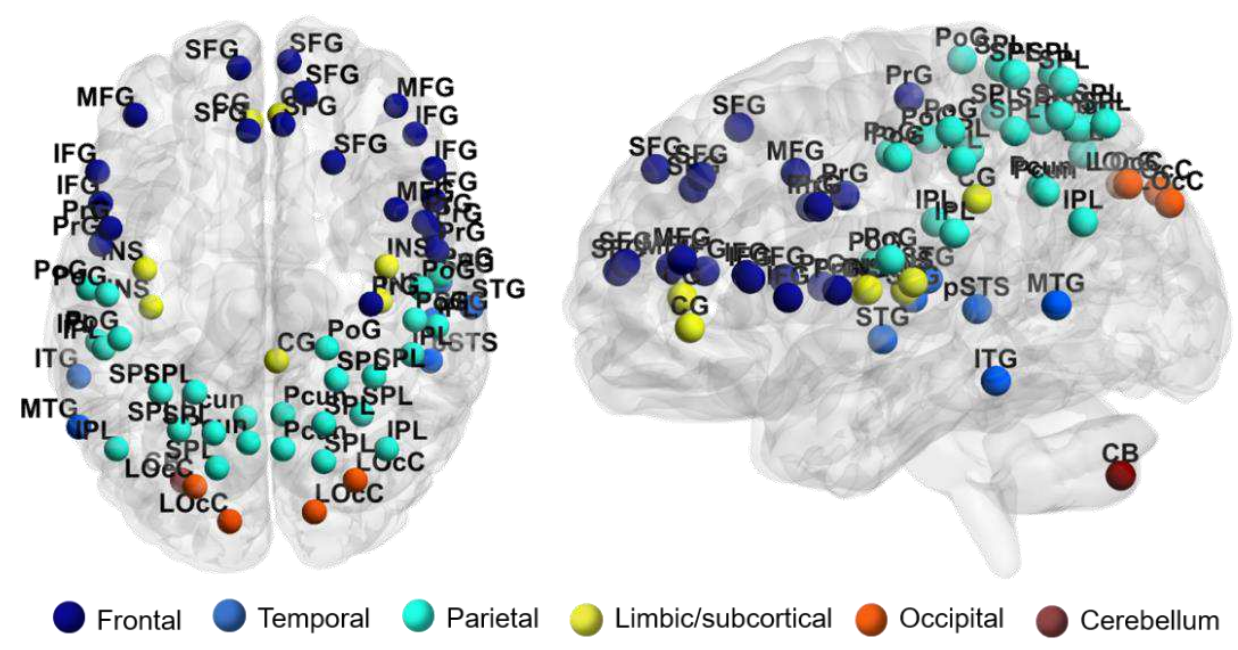

Fig. 3 Nodes showing a significant increase in the children with autism spectrum disorder

81 (A) The local efficiency of the left IFG and cingulate gyrus decreased in the ASD group ( $p<$ 0.05, FDR corrected); (B) The ASD group also showed significantly decreased nodal efficiency in the frontal, parietal, right insula, and visual areas $(p<0.05$, FDR corrected) (ASD group, children with autism spectrum disorder; FDR, false discovery rate; R, right; L, left; MTG, middle temporal gyrus; MFG, middle frontal gyrus; PrG, precentral gyrus; CG, cingulate gyrus; IPL, inferior parietal lobule; IFG, inferior frontal gyrus; SFG, superior

87 frontal gyrus; ITG, inferior temporal gyrus; STG, superior temporal gyrus; STS, superior temporal sulcus; SPL, superior parietal lobule; Pcun, precuneus; PoG, postcentral gyrus; INS, 
89 insula; CB, cerebellum; LOcC, lateraloccipital gyrus) 
To determine whether this underconnectivity observed in our results in the ASD group was due to the sedation effect, we analyzed the group differences in the SLD of the preschool children of both groups subjected to sedation during imaging. In these children (age, 2-6 years) subjected to sedation, overconnectivity was not observed. The functional connectivity between the medial (area 9) and lateral (area 10) parts of the SFG were lower in the ASD group than in the TD group ( $p>0.05$, FDR corrected) (Suppl. Figure 1 and Suppl. Table 2). The connectivity between the right SFG (medial area 9) and right superior parietal lobule (SPL; rostral area 7) also decreased. The right SFG (medial area 10) showed decreased functional connectivity with the left SFG (lateral area 9), right STG (area 41/42 and rostral area 22), right SPL (rostral area 7), right IPL (rostrodorsal area 39), and left IPL (rostroventral 
Discussion

\section{Decreased functional connectivity and impaired social functioning in children with ASD}

This study explored the overall functional connectivity of the brain in relatively young children, aged 2-11 years, with ASD and revealed two major findings. First, the children of the ASD group showed significant alterations as compared to those of the TD group with respect to the functional connectivity of several brain regions included in the DMN, and the global and local/nodal network efficiencies of the ASD children were decreased in these brain areas. Moreover, some of these areas showed significant associations with the clinical symptom scores, such as the CARS, SRS, and ADOS scores. Second, among the preschool-age participants, there was a significant decrease in the functional connectivity in the ASD group. Additionally, in the preschooler participants of the two groups with no difference in the sedation effect, the ASD group showed a marked decrease in the social cognition-related areas, including the DMN.

When comparing the functional connectivity between the groups using network topology based on graph filtration, the ASD group showed decreased connectivity as compared to the TD group in the areas included in the DMN, such as the cingulate gyrus and precuneus (Table 2, Figure 1). DMN dysfunction in ASD has been consistently reported in previous studies (25-27). In a meta-analysis published in 2019, the authors compared eight different studies that analyzed fMRI scans of target groups with various characteristics using several analytical methods of functional connectivity (5). The results of this study showed that as compared to the TD children, the resting state functional connectivity of the DMN regions in the ASD children, such as the dorsal posterior cingulate cortex (PCC) and right medial paracentral lobule, was reduced. None of the studies included in that meta-analysis 
showed an increased local connectivity in ASD children as compared to TD children. In a study involving younger individuals, Yerys et al. (28) compared the resting-state functional connectivity between 22 ASD children aged 8-13 and 22 TD children matched for age, sex, and intelligence quotient. In the ASD children, the functional connectivity of the DMN core regions was reduced in the medial prefrontal cortex and PCC as compared to the TD group. In network segregation analysis, the ASD group showed a pattern of poor segregation of DMN regions as compared to the TD group. Moreover, a significant negative correlation was observed; as the functional connectivity of the DMN decreased, the clinical ASD symptoms worsened.

Similarly, this study found that as the bilateral connectivity between the cingulate gyri decreased in the ASD group, the CARS score, a clinical autism symptom score, increased (Table 2). Considering that the DMN is strongly associated with social cognition $(29,30)$, the association between decreased connectivity of the DMN region and worsening of clinical autism symptoms in the ASD group is reasonable. In addition to the midline structures, we found decreased functional connectivity between the IPL and the frontal and temporal regions (Table 2, Figure 1). The IPL is embedded in several brain networks and converges particularly in the default mode and frontoparietal control networks (31-33). According to previous studies, as a key node of the action observation network, IPL is known to support imitation behavior and may cause difficulties in the social communication function of ASD if impaired (34). Wymbs et al. (35) reported that a decrease in the functional connectivity of the right IPL in children with ASD was associated with impairment of praxis performance and social skills when comparing the fMRI between children aged 8-12 years with ASD and with TD. Although the regions of the brain in our study did not exactly match 
those reported in previous studies, we found that the ASD group showed decreased connectivity as compared to the TD group in several areas linked to the IPL. We also found decreased connectivity between the left IFG and bilateral IPL in the ASD group (Table 2, Figure 1). Another study on imitation in ASD reported that IFG activity was weaker and more delayed in the Asperger syndrome group than in the TD group (36). Furthermore, in a study of the IPL, IFG, and superior temporal sulcus (STS), i.e., brain regions commonly reported to be associated with imitation (36-38), it was found that the effective connectivity between IPL and IFG was reduced in ASD patients. The authors suggested that the aberrant organization of imitation-related networks may be associated with impaired development of social communication in ASD patients (39). In our study, we observed decreased connectivity in the brain regions of IPL, IFG, and STG, which is consistent with the findings of previous studies. Particularly, since the decrease in connectivity between the left IFG and right IPL showed significant correlation with the communication domain of the SRS sub-scores (Table 2), the association between the corresponding brain area and social communication function can be established.

\section{Aberrant network distribution observed in ASD children using graph theoretical measure}

Exploring the difference in the network efficiency between the two groups by applying the graph theoretical method showed that the ASD group had significantly decreased global efficiency as compared to the TD group. Moreover, the local efficiency and nodal efficiency of the ASD group showed decreased network efficiency as compared to the TD group in areas similar to those in the SLD results shown in Table 3 and Figure 3 . As the local efficiency of the left cingulate gyrus decreased, the ADOS total and ADOS social 
interaction scores increased (Table 4). This corroborates with the results identified in the SLD analysis; hence, the importance of the cingulate gyrus in social cognition of ASD is reinforced. The ASD children showed lower nodal efficiencies in several nodes of the frontal, temporal, and parietal areas, bilateral insula, and visual areas as compared to the TD children. On the other hand, the TD group did not show nodal efficiency lower than that of the ASD group. By applying the graph theory, it is possible to identify whether the functional networks of the brain show atypical network distribution, rather than simply distinguishing whether the functional connectivity of ASD patients is over- or underconnected (40). In a previous study, when the graph theoretical analysis was applied to the rs-fMRI of the ASD group and the healthy control group, analysis of global metrics showed significantly lowered clustering coefficient and characteristic path length in the ASD group, indicating that randomized organization was observed in the ASD group when compared with the control group (41). In the same study, significantly altered local metrics were also observed (e.g., the bilateral STS, right dorsolateral prefrontal cortex, and precuneus), and the authors suggested that altered patterns of global and local metrics in ASD may underlie the social and non-social cognitive impairment (41). We found that compared to the control group, the ASD group showed reduced nodal efficiency in brain areas related to social cognition, such as the precuneus, IPL, IFG, STG, and insula (42-44), as well as decreased nodal efficiency in the areas related to non-social cognitive ability in several frontal, temporal, and parietal regions of the brain (4548). Many of the results of nodal efficiency showed a significant correlation with the clinical scores of ADOS, CARS, and SRS, suggesting that the impairment of social/non-social cognitive ability affects the manifestation of the ASD symptoms, as seen in previous studies. 

subjected to sedation with chloral hydrate before imaging. Because we were concerned about medication-induced changes in the functional connectivity, we conducted an additional analysis. Moreover, when children subjected to sedation from the ASD and TD groups were analyzed separately, only preschool children aged 2-6 years were included. Thus, we not only excluded the sedation effect, but also explored the difference in the functional connectivity according to age. When comparing the preschool children under sedation between the two groups, it was observed that the functional connectivity of the DMN area remained significantly reduced in the ASD group (Suppl. Figure 1 and Suppl. Table 2). Particularly, the anterior-posterior DMN connection, such as SFG-IPL and SFG-SPL, was noticeably decreased in children with ASD. The most consistently found aberrant functional connectivity in the ASD group as compared to the TD group is the decreased frontal-posterior functional connectivity pattern. Several previous studies have shown decreased synchronization between the anterior and posterior regions (49). In a study of anatomical and functional connectivity impairment in ASD, Just et al. $(50,51)$ suggested that the behavioral characteristics of ASD are caused by limited communication between the frontal and posterior brain regions. They also predicted that this limitation would affect tasks that require coordination between the frontal and posterior brain regions, such as language comprehension and social interaction processes, and showed that the frontal-posterior coordination was related to these processes based on an fMRI study. Therefore, the authors hypothesized anterior and posterior underconnectivity as the underlying biological mechanism of the impaired function in ASD. Studies on the importance of anterior-posterior coordination in ASD have been conducted 
previously. Horwitz et al. (52) reported that the metabolic activities between several brain regions in male patients with ASD were lower than the normal degree as observed with positron emission tomography. Particularly, they observed that the correlation between the frontal and parietal regions appeared to be reduced. In our study, the decrease in functional connectivity between the frontal and parietal regions observed in the preschool age group included the SFG, a region considered to contribute to higher cognitive function (53), and the SPL and IPL, areas known to be associated with visual motor function $(54,55)$. Furthermore, it has been suggested that the SPL may play an important role in motor learning and repetitive behavior in ASD (56), and the importance of the IPL area is emphasized not only in the control of various movements, but also in the impairment of the social communication function of ASD as mentioned previously (35). Unfortunately, the correlation between the decline in the frontoparietal functional connectivity and the clinical scores in preschool-age children cannot be considered appropriate in this study due to the small sample size. As compared to the TD group, a decrease in the functional connectivity of the right SFG and right STG was also observed in the preschool-age children with ASD. The right STG is known for its important role in social cognition, which is a characteristically impaired function in ASD, and includes theory of mind, biological motion perception, and voice perception (57-59). According to a previous study, the right STG is involved in the detailed perceptual processing of social cognition, which is associated with stronger systematization rather than empathizing, a cognitive characteristic of ASD (60).

Additionally, the functional connectivity between the cerebellar vermis and left IFG decreased in the preschool-age children of the ASD group as compared to the TD group. The cerebellum is one of the brain regions where abnormal findings are most consistently found 
247 in ASD patients (61), and it is extensively connected to the cerebrum; thus, cerebellar

248 dysfunction may be implicated in several symptoms of ASD (62). Particularly, neuropathologic observations of the vermis have been identified from the earliest days of cerebellar imaging studies of ASD (63), and vermal abnormalities are known to be associated with the emotional and behavioral deficits seen in ASD symptoms (64). Previous fMRI studies have reported abnormalities in the vermal activity of individuals with ASD (65). For example, in a previous study, the ASD group showed a decrease in vermal medial lobule activity upon processing of irony (66), while in another study, abnormal recruitment of the posterior cerebellar vermis was observed when ASD participants processed the facial expression (67). The underconnectivity between the cerebellar vermis and frontal region observed in this study is consistent with the findings of previous studies on ASD, suggesting that cerebro-cerebellar connectivity can be a biomarker of ASD. It is known that the integrity of the cerebro-cerebellar circuit in the early development stage is more important for cerebral development than in later development stage (68). Furthermore, the fact that cerebellar damage in childhood can lead to poorer outcomes as compared to that in adulthood $(68,69)$ highlights the significance of the abnormalities of cerebellar functional connectivity found in younger patients with ASD. Recent studies have shown that the integrity of the cerebrocerebellar connectivity is very crucial for early cortical development. As seen in our study, abnormalities in the cerebro-cerebellar functional connectivity found in ASD may impair the development of the cortical areas associated with motor control and language and social interaction, resulting in damage to these domains in ASD patients (65).

\section{Limitations}



functional connectivity of several DMN regions was significantly reduced in the ASD group as compared to the TD group; however, it is possible that sedation effect may affect the results. It is not clear how sedation-induced brain network disruptions might affect fMRI activation patterns (70), and it is possible that sedative agents may affect the fMRI results (71). Therefore, the interpretation of the main results of this study is limited. Although we could exclude the sedation effect from the additional analysis of the preschool age group, it was insufficient because the number of participants was reduced by half. Our findings should be replicated in future studies under conditions that can control the sedation effect. For example, it could be supplemented by developing a method that can conduct imaging in a shorter time without using sedatives. Second, insights regarding the characteristics of the functional connectivity patterns in children with ASD were limited, because the sample size was relatively small. Moreover, the number of participants reduced further when only preschool-age children were considered. The assessment of preschool children in this study is its strength. However, future research should be conducted with more number of participants of the preschool-age group, as well as examination and comparison of the characteristics of functional connectivity patterns between younger and older age groups with ASD. The fMRI results of this study showed the correlation between underconnectivity and clinical scores in several brain regions in the ASD group. By interpreting the corresponding brain regions and clinical symptoms together, the imaging results seemed relevant. However, some of the participants' clinical scores were missing in this study, especially of the SRS scores. and the SRS scores. 


\section{Conclusions}

This study included participants aged $2-11$ years, who were relatively younger than those in previous studies. This study demonstrated the topological network differences using resting state fMRI between the ASD and TD groups, including the main brain networks such as the DMN and social function-related connectivity. We also obtained significant results by exploring the differences in the brain network efficiency using the graph theoretical method. These results showed significant correlations with the clinical scores of the ADOS, CARS, and SRS; thus, the abnormalities in the brain imaging results observed in ASD seem relevant. We conducted additional analyses of the ASD and TD participants of preschool age to exclude the sedation effect from the imaging results. Interestingly, decreased long-range connectivity of more brain regions and decreased cerebro-cerebellar connectivity were observed in the younger participants of the ASD group. These results confirmed that the brain functional connectivity was more impaired in the ASD children than in the TD children. Notably, our findings show that underconnectivity and disrupted efficiency in brain regions primarily related to social function, including the DMN subregions, could be significant biomarkers of childhood ASD.

\section{List of abbreviations}

ASD, Autism spectrum disorder; TD, Typical development; DMN, Default mode network; ROI, 
scale; DTI, Diffusion tensor imaging; IPL, Inferior parietal lobule; SPL, Superior parietal lobule; IFG, Inferior frontal gyrus; SFG, Superior frontal gyrus; STG, Superior temporal gyrus; ITG, Inferior

317 temporal gyrus; PCC, Posterior cingulate cortex; STS, Superior temporal sulcus; rs-fMRI, resting-

\section{Declaration}

Ethics approval and consent to participate

322 Written informed consent was obtained from all parents or guardians, and the children

323 provided verbal assent to participate after sufficient explanation of the study prior to

324 enrollment. All study protocols were approved by the Institutional Review Board of Seoul

325 National University Hospital (No. 2008-116-1150).

326

327

Consent for publication

328 Not applicable

331 The datasets generated and/or analyzed during the current study are not publicly available to

332 protect individual patient privacy, but are available from the corresponding author on 333 reasonable request. 
336 The authors declare that they have no competing interests.

\section{$338 \quad$ Funding}

339 This research was supported by the Bio \& Medical Technology Development Program of the

340 National Research Foundation (NRF) funded by the Korean government (MSIT) (No.

3412020 M3E5D9080787 to B.-N.K) and the Technology Innovation Program (Industrial Strategic

342 Technology Development Program) (20002769, Development of Next Generation Platform for

343 Diagnosis and Therapeutic of Attention Deficit Hyperactivity Disorder and Intellectual 344 Disability based on Big Data) funded by the Ministry of Trade, Industry, and Energy (MOTIE, 345 Korea).

Authors' contributions

NY and HK analyzed and interpreted the data and wrote and edited the manuscript. YH, HL, JIK, JL, CY, SJ, YDA and DL contributed to the design of the study and wrote and edited the manuscript. BK designed the study and prepared the original draft of the manuscript and funding acquisition. All authors read and approved the final manuscript.

Acknowledgments

354 The authors would like to thank the participants and their families for taking part in this 
355 study.

356

357

358

359

360

361

362

363

364

365

366

367

368

369

370

371

372 


\section{References}

375 1. Müller R-A. From loci to networks and back again: anomalies in the study of autism. Annals

376 of the New York Academy of Sciences. 2008;1145:300-15.

377 2. Rudie JD, Brown JA, Beck-Pancer D, Hernandez LM, Dennis EL, Thompson PM, et al. Altered 378 functional and structural brain network organization in autism. Neurolmage: Clinical. 2013;2:79-94.

379 3. Picci G, Gotts SJ, Scherf KS. A theoretical rut: revisiting and critically evaluating the 380 generalized under/over-connectivity hypothesis of autism. Developmental Science. 2016;19(4):52438149.

382 4. Hull JV, Dokovna LB, Jacokes ZJ, Torgerson CM, Irimia A, Van Horn JD. Resting-State 383 Functional Connectivity in Autism Spectrum Disorders: A Review. Frontiers in Psychiatry. 2017;7(205).

$3845 . \quad$ Lau WKW, Leung M-K, Lau BWM. Resting-state abnormalities in Autism Spectrum Disorders:

385 A meta-analysis. Scientific Reports. 2019;9(1):3892.

386 6. Chen $H$, Duan $X$, Liu F, Lu F, Ma X, Zhang $Y$, et al. Multivariate classification of autism 387 spectrum disorder using frequency-specific resting-state functional connectivity-A multi-center study. Progress in Neuro-Psychopharmacology and Biological Psychiatry. 2016;64:1-9.

389 7. Uddin LQ, Supekar K, Lynch CJ, Khouzam A, Phillips J, Feinstein C, et al. Salience Network- 

between the "default mode network" and the "social brain". Frontiers in human neuroscience.

394 2012;6:189-.

395 9. Lee $H$, Kang H, Chung MK, Kim B, Lee DS. Persistent Brain Network Homology From the

10. Ha $\mathrm{S}$, Lee $\mathrm{H}$, Choi $\mathrm{Y}$, Kang $\mathrm{H}$, Jeon $\mathrm{SJ}$, Ryu JH, et al. Maturational delay and asymmetric

information flow of brain connectivity in SHR model of ADHD revealed by topological analysis of

metabolic networks. Scientific Reports. 2020;10(1):3197.

connectivity in a 6-OHDA-induced mouse model of Parkinson's disease examined using persistent

402 homology-based analysis. Scientific reports. 2016;6:33875-.

12. Lord C, Risi S, Lambrecht L, Cook EH, Leventhal BL, DiLavore PC, et al. The Autism Diagnostic

Associated with the Spectrum of Autism. Journal of Autism and Developmental Disorders. 2000;30(3):205-23.

407

13. Eric Schopler MEVB, Glenna Janette Wellman, Steven R. Love. Childhood Autism Rating Test for Korean Children. Korean Educational Development Institute, Seoul1996. 
411 15. Shin MS CS. Korean Leiter international performance scale-revised (K-Leiter-R). Seoul:

412 Hakjisa; 2010.

413 16. Constantino JN. Social Responsiveness Scale. In: Volkmar FR, editor. Encyclopedia of Autism

414 Spectrum Disorders. New York, NY: Springer New York; 2013. p. 2919-29.

415 17. Sparrow SS. Vineland Social Maturity Scales. In: Kreutzer JS, DeLuca J, Caplan B, editors.

416 Encyclopedia of Clinical Neuropsychology. New York, NY: Springer New York; 2011. p. $2621-2$.

417 18. Patel AX, Kundu P, Rubinov M, Jones PS, Vértes PE, Ersche KD, et al. A wavelet method for 418 modeling and despiking motion artifacts from resting-state fMRI time series. Neuroimage. 419 2014;95(100):287-304.

420 19. Fonov V, Evans A, McKinstry R, Almli CR, Collins D. Unbiased nonlinear average age421 appropriate brain templates from birth to adulthood. Neuroimage. 2009;47.

422 20. Fonov V, Evans AC, Botteron K, Almli CR, McKinstry RC, Collins DL, et al. Unbiased average 423 age-appropriate atlases for pediatric studies. Neuroimage. 2011;54(1):313-27.

424 21. Fan L, Li H, Zhuo J, Zhang Y, Wang J, Chen L, et al. The Human Brainnetome Atlas: A New 425 Brain Atlas Based on Connectional Architecture. Cerebral Cortex. 2016;26(8):3508-26.

426 22. Diedrichsen J, Balsters JH, Flavell J, Cussans E, Ramnani N. A probabilistic MR atlas of the 427 human cerebellum. Neuroimage. 2009;46(1):39-46.

428 23. Rubinov M, Sporns O. Complex network measures of brain connectivity: Uses and 429 interpretations. Neuroimage. 2010;52(3):1059-69. 
430 24. Achard S, Bullmore E. Efficiency and cost of economical brain functional networks. PLoS

431 Comput Biol. 2007;3(2):e17.

432 25. Glerean E, Pan RK, Salmi J, Kujala R, Lahnakoski JM, Roine U, et al. Reorganization of 433 functionally connected brain subnetworks in high-functioning autism. Human Brain Mapping. $434 \quad 2016 ; 37(3): 1066-79$.

435 26. He C, Chen Y, Jian T, Chen H, Guo X, Wang J, et al. Dynamic functional connectivity analysis 436 reveals decreased variability of the default-mode network in developing autistic brain. Autism 437 Research. 2018;11(11):1479-93.

438 27. Moseley RL, Ypma RJF, Holt RJ, Floris D, Chura LR, Spencer MD, et al. Whole-brain functional 439 hypoconnectivity as an endophenotype of autism in adolescents. Neurolmage Clinical. 2015;9:14044052.

441 28. Yerys BE, Gordon EM, Abrams DN, Satterthwaite TD, Weinblatt R, Jankowski KF, et al. Default 442 mode network segregation and social deficits in autism spectrum disorder: Evidence from non443 medicated children. Neurolmage: Clinical. 2015;9:223-32.

444 29. Lynch CJ, Uddin LQ, Supekar K, Khouzam A, Phillips J, Menon V. Default mode network in 445 childhood autism: posteromedial cortex heterogeneity and relationship with social deficits. Biol 446 Psychiatry. 2013;74(3):212-9.

447 30. Uddin LQ, lacoboni M, Lange C, Keenan JP. The self and social cognition: the role of cortical 448 midline structures and mirror neurons. Trends in Cognitive Sciences. 2007;11(4):153-7. 
449 31. Andrews-Hanna JR, Reidler JS, Sepulcre J, Poulin R, Buckner RL. Functional-Anatomic 450 Fractionation of the Brain's Default Network. Neuron. 2010;65(4):550-62.

451 32. Buckner RL, Andrews-Hanna JR, Schacter DL. The brain's default network: Anatomy, function, 452 and relevance to disease. The year in cognitive neuroscience 2008. Annals of the New York 453 Academy of Sciences. Malden: Blackwell Publishing; 2008. p. 1-38.

454 33. Parlatini V, Radua J, Dell'Acqua F, Leslie A, Simmons A, Murphy DG, et al. Functional 455 segregation and integration within fronto-parietal networks. Neuroimage. 2017;146:367-75.

456 34. Oberman LM, Ramachandran VS. The simulating social mind: the role of the mirror neuron 457 system and simulation in the social and communicative deficits of autism spectrum disorders. 458 Psychol Bull. 2007;133(2):310-27.

459 35. Wymbs NF, Nebel MB, Ewen JB, Mostofsky SH. Altered Inferior Parietal Functional 460 Connectivity is Correlated with Praxis and Social Skill Performance in Children with Autism Spectrum 461 Disorder. Cereb Cortex. 2021;31(5):2639-52.

462 36. Nishitani N, Avikainen S, Hari R. Abnormal imitation-related cortical activation sequences 463 in Asperger's syndrome. Annals of Neurology. 2004;55(4):558-62.

464 37. Pfeifer JH, lacoboni M, Mazziotta JC, Dapretto M. Mirroring others' emotions relates to 465 empathy and interpersonal competence in children. Neuroimage. 2008;39(4):2076-85.

466 38. Williams JHG, Waiter GD, Gilchrist A, Perrett DI, Murray AD, Whiten A. Neural mechanisms 467 of imitation and 'mirror neuron' functioning in autistic spectrum disorder. Neuropsychologia. 
39. Shih P, Shen M, Öttl B, Keehn B, Gaffrey MS, Müller R-A. Atypical network connectivity for 470 imitation in autism spectrum disorder. Neuropsychologia. 2010;48(10):2931-9.

471 40. Keown $C L$, Datko $M C$, Chen $C P$, Maximo JO, Jahedi A, Müller R-A. Network Organization Is 472 Globally Atypical in Autism: A Graph Theory Study of Intrinsic Functional Connectivity. Biological Psychiatry: Cognitive Neuroscience and Neuroimaging. 2017;2(1):66-75.

474 41. Itahashi T, Yamada T, Watanabe H, Nakamura M, Jimbo D, Shioda S, et al. Altered Network 475 Topologies and Hub Organization in Adults with Autism: A Resting-State fMRI Study. PLOS ONE. 2014;9(4):e94115. 42. Hadjikhani N, Joseph RM, Snyder J, Tager-Flusberg H. Anatomical Differences in the Mirror 478 Neuron System and Social Cognition Network in Autism. Cerebral Cortex. 2006;16(9):1276-82.

479 43. Odriozola $P$, Uddin LQ, Lynch CJ, Kochalka J, Chen T, Menon V. Insula response and connectivity during social and non-social attention in children with autism. Social cognitive and affective neuroscience. 2016;11(3):433-44. 44. Patriquin MA, DeRamus $T$, Libero LE, Laird A, Kana RK. Neuroanatomical and neurofunctional markers of social cognition in autism spectrum disorder. Human Brain Mapping. 2016;37(11):3957-78.

45. Kana RK, Keller TA, Minshew NJ, Just MA. Inhibitory Control in High-Functioning Autism: 
46. Lukito S, Norman L, Carlisi C, Radua J, Hart H, Simonoff E, et al. Comparative meta-analyses of brain structural and functional abnormalities during cognitive control in attentiondeficit/hyperactivity disorder and autism spectrum disorder. Psychological medicine. 2020;50(6):894-

47. Schmitz N, Rubia K, Daly E, Smith A, Williams S, Murphy DGM. Neural Correlates of

493 Executive Function in Autistic Spectrum Disorders. Biological Psychiatry. 2006;59(1):7-16.

494 48. Shafritz KM, Dichter GS, Baranek GT, Belger A. The Neural Circuitry Mediating Shifts in 495 Behavioral Response and Cognitive Set in Autism. Biological Psychiatry. 2008;63(10):974-80.

496 49. Schipul SE, Keller TA, Just MA. Inter-regional brain communication and its disturbance in 497 autism. Frontiers in systems neuroscience. 2011;5:10-.

498 50. Just MA, Cherkassky VL, Keller TA, Minshew NJ. Cortical activation and synchronization during sentence comprehension in high-functioning autism: evidence of underconnectivity. Brain. 2004;127(8):1811-21.

501 51. Just MA, Cherkassky VL, Keller TA, Kana RK, Minshew NJ. Functional and anatomical cortical underconnectivity in autism: evidence from an FMRI study of an executive function task and corpus callosum morphometry. Cereb Cortex. 2007;17(4):951-61.

504 52. Horwitz B, Rumsey JM, Grady CL, Rapoport SI. The Cerebral Metabolic Landscape in Autism: Intercorrelations of Regional Glucose Utilization. Archives of Neurology. 1988;45(7):749-55. 
507 of the left superior frontal gyrus in humans: a lesion study. Brain. 2006;129(12):3315-28.

50854 Fogassi L, Luppino G. Motor functions of the parietal lobe. Current Opinion in Neurobiology. 509 2005;15(6):626-31.

510 55. Goodale MA, Milner AD. Separate visual pathways for perception and action. Trends

511 Neurosci. 1992;15(1):20-5.

512 56. Travers BG, Kana RK, Klinger LG, Klein CL, Klinger MR. Motor Learning in Individuals With

513 Autism Spectrum Disorder: Activation in Superior Parietal Lobule Related to Learning and Repetitive

514 Behaviors. Autism Research. 2015;8(1):38-51.

515 57. Baron-Cohen S, Belmonte MK. AUTISM: A Window Onto the Development of the Social 516 and the Analytic Brain. Annual Review of Neuroscience. 2005;28(1):109-26.

517 58. Beauchamp MS. The social mysteries of the superior temporal sulcus. Trends in Cognitive 518 Sciences. 2015;19(9):489-90.

519 59. Pelphrey KA, Carter EJ. Charting the typical and atypical development of the social brain.

520 Development and Psychopathology. 2008;20(4):1081-102.

521 60. Kobayashi A, Yokota S, Takeuchi H, Asano K, Asano M, Sassa Y, et al. Increased grey matter 522 volume of the right superior temporal gyrus in healthy children with autistic cognitive style: A VBM 523 study. Brain and Cognition. 2020;139:105514.

524 61. Fatemi SH, Aldinger KA, Ashwood P, Bauman ML, Blaha CD, Blatt GJ, et al. Consensus paper: 
525 pathological role of the cerebellum in autism. Cerebellum (London, England). 2012;11(3):777-807.

526 62. Rogers T, McKimm E, Dickson P, Goldowitz D, Blaha C, Mittleman G. Is autism a disease of 527 the cerebellum? An integration of clinical and pre-clinical research. Frontiers in Systems 528 Neuroscience. 2013;7(15).

529 63. Courchesne E, Yeung-Courchesne R, Press GA, Hesselink JR, Jernigan TL. Hypoplasia of 530 cerebellar vermal lobules VI and VII in autism. N Engl J Med. 1988;318(21):1349-54.

531 64. Tavano A, Grasso R, Gagliardi C, Triulzi F, Bresolin N, Fabbro F, et al. Disorders of cognitive 532 and affective development in cerebellar malformations. Brain. 2007;130(10):2646-60.

533 65. D'Mello AM, Stoodley CJ. Cerebro-cerebellar circuits in autism spectrum disorder. Frontiers 534 in Neuroscience. 2015;9(408).

535 66. Wang AT, Lee SS, Sigman M, Dapretto M. Reading affect in the face and voice: neural 536 correlates of interpreting communicative intent in children and adolescents with autism spectrum 537 disorders. Arch Gen Psychiatry. 2007;64(6):698-708.

538 67. Critchley HD, Daly EM, Bullmore ET, Williams SCR, Van Amelsvoort T, Robertson DM, et al.

539 The functional neuroanatomy of social behaviour: Changes in cerebral blood flow when people with

540 autistic disorder process facial expressions. Brain. 2000;123(11):2203-12.

541 68. Wang $\mathrm{SSH}$, Kloth $\mathrm{AD}$, Badura A. The cerebellum, sensitive periods, and autism. Neuron. $542 \quad 2014 ; 83(3): 518-32$.

543 69. Scott RB, Stoodley CJ, Anslow P, Paul C, Stein JF, Sugden EM, et al. Lateralized cognitive 
544 deficits in children following cerebellar lesions. Dev Med Child Neurol. 2001;43(10):685-91.

545 70. Di Francesco MW, Robertson SA, Karunanayaka P, Holland SK. BOLD fMRI in infants under

546 sedation: Comparing the impact of pentobarbital and propofol on auditory and language activation.

547 Journal of Magnetic Resonance Imaging. 2013;38(5):1184-95.

548 71. Liu X, Lauer KK, Douglas Ward B, Roberts C, Liu S, Gollapudy S, et al. Propofol attenuates 549 low-frequency fluctuations of resting-state fMRI BOLD signal in the anterior frontal cortex upon loss 550 of consciousness. Neuroimage. 2017;147:295-301. 
562 Additional file 1: DOC. Supplementary Table 1. Region of interest.

563 Additional file 2: DOC. Supplementary Table 2. Edges showing significantly decreased

564 functional connectivity in preschool children with autism spectrum disorder.

565 Additional file 3: DOC. Supplementary Fig. 1. Group differences between the preschool 566 children (age, 2-6 years) of the autism spectrum disorder group and typical development 567 group.

568 Additional file 4: DOC. Supplementary Fig. 2. Distribution of the correlation coefficients. 


\section{Supplementary Files}

This is a list of supplementary files associated with this preprint. Click to download.

- Additionalfile1.docx

- Additionalfile2.docx

- Additionalfile3.docx

- Additionalfile4.docx 